\title{
Avaliação do Compartilhamento Espectral em Redes Cognitivas utilizando Jogos Evolucionários
}

\author{
Marcel W. R. da Silva, Kleber V. Cardoso, David F. C. Moura, \\ Juraci F. Galdino, Ronaldo M. Salles, José Ferreira de Rezende
}

\begin{abstract}
Resumo-Os rádios cognitivos podem representar a solução para o problema da atual utilização ineficiente do espectro de freqüências. Estes dispositivos, capazes de detectar a presença de rádios primários e adaptar suas características de operação, conseguem determinar faixas sem uso ou subutilizadas como oportunidades e utilizá-las para atender às necessidades dos seus usuários. Um dos problemas em aberto no desenvolvimento destes rádios é a coordenação do compartilhamento espectral entre eles. Quando duas ou mais redes cognitivas operam na mesma região, o compartilhamento espectral alcançado afeta diretamente o seu desempenho. Tomando os rádios cognitivos como dispositivos inteligentes que possuem interesses conflitantes, propomos um modelo para a análise do problema de compartilhamento de espectro entre redes cognitivas utilizando Teoria dos Jogos Evolucionária e discutimos as vantagens e desvantagens da modelagem do comportamento dos jogadores através de uma Cadeia de Markov e de um Algoritmo Genético.
\end{abstract}

Palavras-Chave-Rádios Cognitivos, Teoria dos Jogos.

Abstract-Cognitive radios may be a great solution to the inefficient spectrum use problem present nowadays. Those devices are capable of detecting and exploiting unused spectrum bands in licensed portions of spectrum to attend to their users needs. A major open issue in cognitive or secondary radios and networks design is spectrum sharing between them. When more than two radios operate in the same region, spectrum sharing may pose problems in their performance. Considering cognitive radios as intelligent devices with conflicting interests, we propose a model to analyze spectrum sharing problem using evolutionary game theoretical framework. Furthermore, we evaluate the pros and cons of two player's behavior modeling techniques: using Markov Chains and Genetics Algorithms.

Keywords - Cognitive Radios, Game theory.

\section{INTRODUÇÃO}

Nos últimos anos o interesse da comunidade científica a respeito dos Rádios Cognitivos aumentou significativamente. Esse interesse deve-se ao fato de que tais dispositivos podem representar uma solução eficiente para o problema da má utilização do espectro de freqüências existente atualmente.

As agências regulamentadoras dividem o espectro de freqüências em faixas licenciadas e não-licenciadas. No espectro licenciado, o qual representa a maior parte das freqüências, podem operar apenas dispositivos de uma determinada tecnologia que adquirem uma licença de uso daquela parte do espectro naquela região. Entretanto, em uma determinada região podem não existir dispositivos em uma faixa licenciada, ou estes dispositivos quando presentes podem ocupar o espectro

Marcel W. R. da Silva, Kleber V. Cardoso e José Ferreira de Rezende, Grupo de Teleinformática e Automação (GTA), Programa de Engenharia Elétrica (PEE), COPPE/UFRJ, Rio de Janeiro, Brasil, E-mails: \{marcel, kleber, rezende\}@gta.ufrj.br. David F. C. Moura, Juraci F. Galdino, Ronaldo M. Salles, Instituto Militar de Engenharia (IME), Rio de Janeiro, Brasil, Emails: dfcmoura@uol.com.br, \{galdino,salles\}@ime.eb.br. apenas periodicamente [7], [2], [3], [6], [11], [1]. Enquanto isso, as faixas de freqüências não-licenciadas tornam-se cada vez mais ocupadas, fazendo os dispositivos sem fio sofrerem com a limitação de capacidade e os problemas de interferência.

Dentro do contexto apresentado, os rádios cognitivos podem representar grandes avanços, pois conseguem detectar e aprender com as limitações impostas pelo meio de comunicação, como a presença de interferência indesejada ou de outros dispositivos licenciados. Dessa forma, um rádio cognitivo pode adaptar suas características, como freqüência de operação, largura de banda, potência de transmissão ou modulação, para aprimorar seu desempenho visando alcançar a capacidade requerida pelas aplicações de seus usuários.

Por outro lado, vários dispositivos cognitivos (rádios ou redes) podem compartilhar o mesmo espectro e cada equipamento pode realizar escolhas distintas de seus parâmetros de operação, buscando alcançar a capacidade que deseja [1]. Surgem então dúvidas sobre o comportamento de uma população de rádios cognitivos sem qualquer tipo de controle, seja ele centralizado ou distribuído. Afinal, qual será a capacidade que cada rádio conseguirá obter? Quais escolhas prevalecem na configuração das características de operação? Baseados apenas no que observam individualmente do espectro, os rádios conseguem realizar as melhores escolhas ou outras decisões trariam maior ganho a todos? Para responder a perguntas como essas, é utilizada a Teoria dos Jogos no problema de compartilhamento do espectro por rádios cognitivos.

Em especial, a Teoria dos Jogos Evolucionária é escolhida por oferecer maior flexibilidade que a Teoria Clássica. Porém, rádios cognitivos introduzem uma dificuldade adicional, a quantidade de características de um equipamento é alta. Isso faz com que os modelos matemáticos baseados em estados (como a cadeia de Markov) se tornem restritivos, pois o número de estados aumenta significativamente. Para conseguir manter o problema tratável, sem restringir excessivamente a representação matemática do mesmo, são utilizados Algoritmos Genéticos. A resolução do jogo deixa de ser uma questão de encontrar o estado estacionário de uma cadeia ergódica, passando para a descoberta de pontos ótimos em funções multi-objetivo. Como será apresentado posteriormente, essa abordagem inovadora traz ganhos satisfatórios na escalabilidade, sem perdas na acurácia.

Esse artigo está organizado da seguinte forma. Na Seção II, são apresentados os trabalhos relacionados. Já na Seção III, é feita uma breve descrição dos conceitos principais envolvendo rádios cognitivos e teoria de jogos. A Seção IV apresenta um cenário de aplicação para os rádios cognitivos e introduz o modelo matemático usado para descrição do jogo. A Seção V mostra como o modelo é utilizado, a resolução do jogo e 
responde perguntas importantes sobre o comportamento da população de rádios cognitivos (ou jogadores). A Seção VI conclui o trabalho e discute perspectivas a serem exploradas.

\section{TRABAlhos RELACIONADOS}

Muitos trabalhos foram publicados a respeito de rádios cognitivos. Dentre eles, [6] e [1] se destacam por apresentarem o problema de maneira mais geral, buscando levantar os aspectos mais importantes para o seu desenvolvimento. Além disso, [1] propõe uma classificação dos problemas em aberto em rádios cognitivos de acordo com suas funcionalidades. Dentre eles, destaca-se o problema do compartilhamento do espectro entre os rádios ou redes cognitivas que é o objeto de estudo deste artigo.

Alguns trabalhos recentes têm abordado o problema do compartilhamento espectral em redes cognitivas usando teoria dos jogos. Em [10] os autores consideram em seu modelo que a atividade dos rádios cognitivos em uma banda licenciada será negociada com os dispositivos primários presentes naquela região. Com isso, [10] propõem um modelo desta interação entre dispositivos primários e secundários como um jogo de Cournot, utilizado em economia para avaliar a interação entre empresas (rádios cognitivos) que disputam pelo aumento de seu lucro (quantidade de banda) perante um mercado (dispositivos primários).

Em [5], é proposto um modelo simples onde os jogadores disputam os canais livres em um cenário com dispositivos primários. A recompensa dos jogadores é a taxa máxima que pode ser obtida de acordo com a disponibilidade dos canais e quantidade de interferência. Além disso, considera-se que os rádios secundários usam um método de acesso ao meio CSMA persistente. Os autores avaliam o impacto da existência de jogadores mais ou menos agressivos no resultado do equilíbrio atingido pelo jogo.

Em [9], os autores apresentam um modelo para o compartilhamento de espectro entre rádios cognitivos distribuídos em uma determinada região, utilizando um jogo potencial exato. Nesse modelo, a potência e a largura de banda são fixas e os jogadores são capazes de ajustar sua modulação de acordo com a relação sinal-ruído (Signal to Interference plus Noise Ratio - SINR) experimentada, tendo como base os valores de SINR alvo para cada tipo de modulação. A partir do modelo, os autores propõem e avaliam um mecanismo simples de coordenação entre os rádios cognitivos que opera nos moldes do modelo desenvolvido em jogos potenciais.

Baseado no uso de teoria dos jogos será apresentado uma nova modelagem do problema do compartilhamento do espectro entre rádios cognitivos utilizando jogos evolucionários. A grande vantagem deste tipo de jogo é a sua flexibilidade no que diz respeito à modelagem do comportamento dos jogadores.

\section{TEORIA DOS JOGOS}

A Teoria dos Jogos é uma ferramenta matemática para ser utilizada na representação e análise de situações de conflito entre dois ou mais jogadores. Normalmente, o conflito entre os jogadores surge pela disputa por um recurso compartilhado e insuficiente, por exemplo o espectro de transmissão. Nesse contexto, a teoria dos jogos ajuda a entender como os jogadores vão se comportar, ou seja, quais estratégias eles vão escolher. A escolha de uma estratégia por um jogador está ligada à recompensa (payoff) que o mesmo recebe, geralmente descrita de forma numérica em uma função utilidade.

A estratégia escolhida por cada jogador e a recompensa que cada um obtem definem um estado do jogo. Alguns estados do jogo são especiais, pois representam propriedades importantes da dinâmica entre os jogadores. Um desses estados é o Equilíbrio de Nash, o qual descreve um estado do jogo onde nenhum jogador consegue melhorar sua recompensa alterando sua estratégia unilateralmente. Outro estado importante é o Ótimo de Pareto, o qual representa um estado no qual não há nenhum outro resultado do jogo que ofereça a todos os jogadores uma recompensa melhor.

A Teoria dos Jogos Clássica apresenta uma grande variedade de tipos de jogos que podem ser úteis para representar diferentes tipos de conflito. No entanto, os tipos de jogos disponíveis apresentam algumas restrições e, portanto, será utilizada a Teoria dos Jogos Evolucionária para modelar a disputa do espectro sem fio pelos rádios cognitivos.

\section{A. Jogos Evolucionários}

Um acréscimo importante da Teoria dos Jogos Evolucionária é o tratamento dos jogadores como agentes capazes aprender, imitar uns aos outros e experimentar usando tentativa e erro. Assim, essa nova teoria é capaz modelar sistemas dinâmicos, ou seja, que evoluem com o tempo. A proporção da população dos jogadores adotando uma determinada estratégia é um elemento importante em jogos evolucionários, pois essa informação é parte da dinâmica do jogo e participa da determinação do resultado do mesmo.

Em jogos evolucionários, um conceito importante é a Estratégia Evolucionariamente Estável (Evolutionary Stable Strategy - ESS), a qual define um estado do jogo onde uma população adotando estratégias segundo uma distribuição não é vulnerável a invasões por indivíduos que possam alterar tal distribuição. Se um estado é ESS, então ele também é um equilíbrio de Nash.

Além disso, na teoria dos jogos evolucionários, os jogadores podem pertencer a "classes" diferentes, possuir conjuntos de estratégias diferentes e funções utilidade assimétricas, oferecendo grande flexibilidade na representação de problemas. No entanto, vários cenários podem ser descritos por um jogo evolucionário mais simples, no qual os jogadores são do mesmo tipo, utilizam estratégias idênticas e têm funções utilidade simétricas.

\section{Estudo de CASO}

Rádios cognitivos constituem uma tecnologia nova, ainda sendo predominante em laboratórios de pesquisa. No entanto, os mesmos já possuem aplicações potenciais de grande interesse militar e comercial. Nessa seção, é ilustrado um cenário potencial para uso comercial dos rádios cognitivos em uma provedora de serviço de telecomunicações. $\mathrm{O}$ ambiente descrito também é utilizado para estabelecer as características que se deseja representar no modelo matemático do jogo 


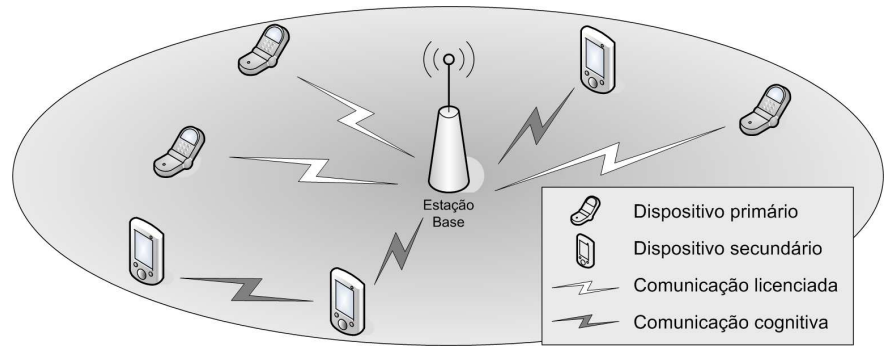

Fig. 1. Dispositivos primários e secundários compartilhando o espectro

evolucionário, ou seja, definir jogadores, estratégias, função utilidade, entre outros.

\section{A. Cenário de Aplicação}

Um cenário simples de aplicação de rádios cognitivos é o uso como aparelhos móveis celulares. Dessa forma, uma operadora de telefonia celular conseguiria aumentar a eficiência na utilização da banda que tem concessão. Neste contexto, a operadora poderia disponibilizar aos clientes dispositivos cognitivos que utilizassem de maneira oportunista as faixas de espectro não utilizadas pelos clientes primários (Figura 1). Esse serviço não afetaria o desempenho dos usuários primários e poderia ser disponibilizado a um menor custo para os clientes portadores de tais dispositivos secundários.

Os dispositivos secundários (rádios cognitivos) são capazes de detectar (ou ser informados) sobre os outros rádios primários presentes em uma região. Assim, eles adaptam suas características para atender as demandas dos seus usuários e ao mesmo tempo não prejudicar os usuários primários. $\mathrm{O}$ usuário de um dispositivo secundário nessas condições estaria disposto a abrir mão de parte da qualidade em função de um menor custo. No entanto, o usuário apenas teria interesse nesse tipo de serviço se soubesse quais são os limites inferiores de qualidade que ele vai encontrar.

No contexto descrito, um dos problemas de interesse para a operadora é entender como ocorre o compartilhamento das oportunidades entre estes dispositivos secundários. Uma vez que tais dispositivos secundários têm o objetivo de atender às demandas dos seus usuários, podem existir cenários onde a sua demanda agregada não poderá ser atendida pelas oportunidades de espectro disponíveis. Dessa forma, podemos visualizar os usuários secundários como indivíduos com interesses conflitantes, onde, ao tentar suprir sua demanda, os indivíduos prejudicam o desempenho uns dos outros devido ao aumento da interferência. Ou seja, um cenário típico para aplicação de teoria dos jogos, a qual permite modelar o comportamento destes indivíduos e determinar qual seria sua situação de equilíbrio. Além disso, podemos estudar também as características deste ponto de equilíbrio e determinar se ele atende aos interesses da operadora ao mesmo tempo em que supre as necessidades dos usuários. Poderíamos dizer ainda se há outra situação que atende melhor a todos, ainda que a mesma não seja de equilíbrio.

Para representar o problema proposto será usado um jogo do tipo evolucionário, descrito na Seção III-A. Esse tipo de jogo permite uma maior flexibilidade no que diz respeito

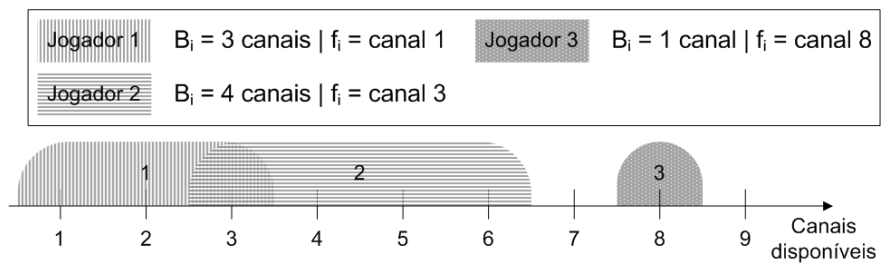

Fig. 2. Exemplo de sobreposição espectral entre dois jogadores

à modelagem do comportamento dinâmico dos jogadores. Dessa forma, podemos levar em consideração as consecutivas interações entre os jogadores (os rádios cognitivos), os quais são dispositivos "inteligentes" e que aprendem com as suas experiências passadas. A próxima seção apresenta maiores detalhes deste modelo do problema.

\section{B. Modelo do Sistema}

Para modelar o compartilhamento de recursos entre rádios cognitivos utilizaremos o jogo evolucionário $J$. Nesse jogo, vamos considerar que há $N$ jogadores (rádios cognitivos) disputando as oportunidades do espectro de uma determinada operadora. No nosso modelo, os dispositivos secundários não podem operar nos canais onde existem rádios primários. A influência dos usuários primários é representada então como o limitante para a quantidade de oportunidades disponíveis.

Os jogadores têm disponíveis uma determinada quantidade de canais $C$. Para usufruir da capacidade dos $C$ canais, os jogadores contam com uma cesta de estratégias flexível, representada pela quádrupla $E_{i}=<P_{i}, B_{i}, M_{i}, f_{i}>$, onde $P_{i}$ é a potência de transmissão, $B_{i}$ é a banda utilizada dada por um número inteiro representando o número de canais utilizados, $M_{i}$ é o número de símbolos da modulação e $f_{i}$ é a freqüência de transmissão representada pelo número do canal mais a esquerda da banda $B_{i}$. Para cada um desses quatro parâmetros, é especificado um conjunto finito de opções.

Em nosso modelo, o conflito entre os jogadores é representado pela interferência mútua gerada quando dois ou mais jogadores escolhem estratégias conflitantes. Dependendo, por exemplo, da largura de banda $B_{i}$ e da freqüência de operação $f_{i}$, pode existir sobreposição espectral entre os sinais de dois ou mais jogadores. Para descrever esse efeito, vamos considerar que uma porcentagem da potência (proporcional à porcentagem de sobreposição espectral) utilizada por um jogador é recebida como ruído pelo outro jogador com estratégia "sobreposta". A Figura 2 exibe um cenário onde três jogadores compartilham canais do espectro. Neste exemplo, os jogadores 1 e 2 escolhem estratégias onde seus canais apresentam sobreposição espectral. Como eles possuem um canal sobreposto, o jogador 1 recebe $25 \%$ da potência gerada pelo jogador 2 como interferência e o jogador 2 recebe $33 \%$ da potência do jogador 1 como interferência.

Além do fenômeno da sobreposição espectral, a distância entre os transmissores também possui um papel importante na atenuação dos sinais interferentes. No nosso modelo, adicionamos um parâmetro de atenuação $L_{(i, j)}$ responsável por escalonar a potência dos sinais interferentes gerados pelo jogador $i$ e recebidos pelo jogador $j$. Por questões didáticas, consideramos que $L_{(i, j)}$ é uma constante $L$ para todo par $(i, j)$. 


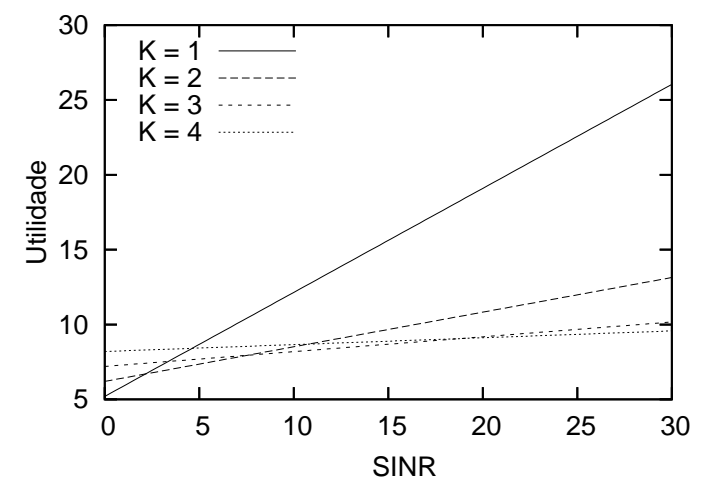

Fig. 3. Função utilidade de acordo com a $S I N R$ e $K$

\section{Função Utilidade}

Uma vez estabelecidos os jogadores e suas estratégias, é necessário descrever o que os motiva durante o jogo, ou seja, a sua recompensa. Em um jogo evolucionário é a função utilidade que determina a recompensa de um jogador em cada estágio (round) do jogo. Para modelar esta grandeza utilizamos a probabilidade de erro de bit (Bit Error Rate - BER) e a eficiência espectral $(K)$ de acordo com a equação 1 . Na função proposta pode-se perceber a presença de valores alvo para a eficiência espectral e a BER, representando limiares mínimos para garantir uma determinada qualidade de serviço (Quality of Service - QoS) ao jogador $i$. Ou seja, a utilidade $\left(u_{i}\right)$ representa o nível de satisfação do jogador $i$ de acordo com a sua distância dos limiares de QoS para eficiência espectral $\left(K_{\text {alvo }}\right)$ e BER $\left(B E R_{\text {alvo }}\right)$. A constante $a$ presente na fórmula entra apenas como um fator de ajuste, e seu valor é escolhido de forma que a utilidade não assuma valores negativos.

$$
u_{i}(.)=\left(K_{i}-K_{\text {alvo }}\right)-\log _{10}\left(B E R_{i} / B E R_{\text {alvo }}\right)+a
$$

Para o cálculo da BER em cada tipo de modulação, utilizamos a equação apresentada em (2) para modulação do tipo M-QAM. Ela é uma aproximação que possui a vantagem de ser muito mais simples que a equação original e apresentar uma boa estimativa. Nela, $K_{i}$ é a eficiência espectral obtida de acordo com robustez da modulação escolhida (equação 3 ).

$$
\begin{gathered}
B E R_{M-Q A M}=0.2 \exp \frac{-1.6 S I N R}{2^{K_{i}}-1} \\
K_{i}\left(M_{i}\right)=\log _{2}\left(M_{i}\right)
\end{gathered}
$$

A Figura 3 exibe o gráfico da função utilidade observada por um jogador de acordo com a SINR e de acordo com a eficiência espectral $K$ experimentada. Para estes gráficos utilizamos $K_{\text {alvo }}=4,5 \mathrm{bps} / \mathrm{hertz}$ e $B E R_{\text {alvo }}=10^{-2}$, valores típicos desejáveis para uma transmissão de voz. Ao mudar de estratégia, alterando a largura de banda $B_{i}$, a potência $P_{i}$ ou a freqüências de operação $f_{i}$ este jogador sofre uma variação da SINR devido à mudança na interferência e na potência de recepção. Ao alterar o tipo de modulação $M_{i}$ provoca-se uma variação na eficiência espectral.

Pelo resultado apresentado na Figura 3, é possível perceber que, apesar de apresentar coerência física, a função utilidade

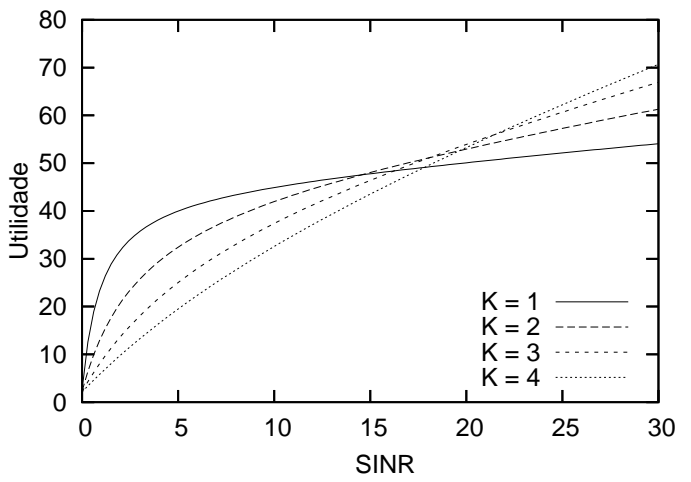

Fig. 4. Nova função utilidade de acordo com o $S I N R$ e $K$

(1) não representa da maneira desejada o nível de satisfação do jogador. Para valores baixos de SINR, a utilidade aumentou com o incremento de $K$, tendo um comportamento inverso para valores altos de SINR. Seria esperado, o contrário, pois para valores altos de SINR, se torna viável usar valores mais altos de $K$ (ou seja, maior número de bits por símbolo). Isto ocorre porque o aumento da SINR deveria permitir o jogador operar com uma modulação mais complexa sem aumentar muito sua $B E R$.

Com intuito de obter uma melhor representação do nível de satisfação dos jogadores de acordo com as estratégias escolhidas, definimos uma nova função utilidade representada pela equação 4. Nessa nova abordagem, alteramos o grau de influência da SINR e da eficiência espectral para descrever melhor a recompensa dos jogadores na escolha de uma estratégia. A Figura 4 exibe o gráfico da nova função utilidade.

$$
u_{i}(.)=\frac{\left(K_{i} S I N R_{i}\right)}{3}+\frac{1}{\left(K_{i}^{2}\right)}+\frac{30}{\log 10\left(B E R_{i}\right)}+a
$$

Como pode se observar, as imperfeições da equação anterior foram removidas. Além disso, está função possui todas as características desejáveis para a representação da satisfação de um jogador no problema que desejamos avaliar. Quando a SINR é baixa, mais vale utilizar uma modulação mais robusta a interferência que outras mais complexas. Em contrapartida, com o aumento da SINR o uso das modulações mais complexas, que fornecem melhores taxas de transmissão, se torna cada vez mais satisfatório.

Para um jogo evolucionário, uma característica importante é a dinâmica do comportamento dos jogadores. Na Seção V, apresentamos o uso de dois tipos de modelagem, uma com cadeias de Markov e outra com algoritmos genéticos.

\section{Algoritmos Genéticos}

Como forma de comparar os resultados e a eficiência com trabalhos disponíveis na literatura, além do modelo utilizando cadeias de Markov, elaborou-se um simulador empregando algoritmos genéticos para representar as estratégias evolucionariamente estáveis.

$\mathrm{Na}$ simulação com algoritmos genéticos, desenvolvida com base no algoritmo genético simples descrito em [4], há uma população inicial gerada aleatoriamente. Cada indivíduo 


\begin{tabular}{|c||c|}
\hline \multicolumn{1}{|c|}{ Métrica } & Valor \\
\hline \hline Tamanho da População & 20 ou 120 (2 ou 3/4 jogadores) \\
\hline Número de Gerações & 20 ou 120 (2 ou 3/4 jogadores $)$ \\
\hline Probabilidade de Cruzamento & $x \in[0.2,0.3]$ \\
\hline Probabilidade de Mutação & 0.0125 \\
\hline
\end{tabular}

TABELA I

PARÂMETros do Algoritmo GenÉtico

da população (o cromossomo) representa a combinação de estratégias adotadas pelos jogadores presentes no sistema. As estratégias de cada jogador são descritas por 5 genes, representados de forma binária, de forma a descrever completamente as 20 combinações possíveis de largura de banda/potência/freqüência/modulação. Assim, dados $n$ jogadores, cada cromossomo é descrito por $5 n$ bits. Em função das escolhas dos $n$ jogadores, cada cromossomo apresenta um valor distinto de função de aptidão.

Em cada geração, o pareamento entre indivíduos é realizado de forma aleatória, adotando o mecanismo de roleta como método de seleção. São também utilizados os operadores de cruzamento entre indivíduos (crossing-over) e de mutação. Estes operadores são aplicados de forma aleatória, de acordo com probabilidades estabelecidas a priori. Os parâmetros de configuração do simulador, como tamanho da população inicial, número de gerações e as probabilidades dos operadores genéticos estão descritos na tabela I.

O algoritmo genético é uma ferramenta poderosa para resolução de funções multi-objetivo, as quais são muitas vezes um problema de alto custo computacional. Para utilizar algoritmos genéticos na descoberta dos equilíbrios de Nash e dos ótimos de Pareto, foi necessário transformar esses estados do jogo em uma função a ser otimizada pelo algoritmo. No entanto, verificamos que, dependendo do modelo do jogo, nem sempre é possível encontrar uma função que represente tais equilíbrios. Para o nosso modelo, o uso da função objetivo 5 descreve os ótimos de Pareto do jogo.

$$
\text { Objetivo }=\sum_{j=1}^{N} \frac{K_{i} * S I N R_{i}}{3}+\frac{1}{2^{K_{i}}}+\frac{30}{\log _{10} B E R_{i}}+45
$$

\section{RESUltados}

Uma qualidade importante dos jogos evolucionários é a maior flexibilidade para modelar o comportamento dos jogadores, como foi apresentado na Seção III-A. Isto nos permite modelar jogadores inteligentes e capazes de aprender com suas experiências passadas. Por outro lado, como outros tipos de jogos, é necessário encontrar determinados estados que possuem propriedades importantes, como equilíbrios de Nash e ótimos de Pareto. Nesse trabalho, avaliamos duas ferramentas para modelar o jogo e encontrar esses estados: Cadeias de Markov (CM) e Algoritmos Genéticos (AG).

Para avaliar as duas abordagens na modelagem do comportamento dos jogadores, definimos inicialmente um cenário básico. Estão disponíveis 3 canais $(l=3)$ para os rádios secundários com uma largura de banda de 22.0 MHz. Duas escolhas para largura de banda são permitidas, estreita (1

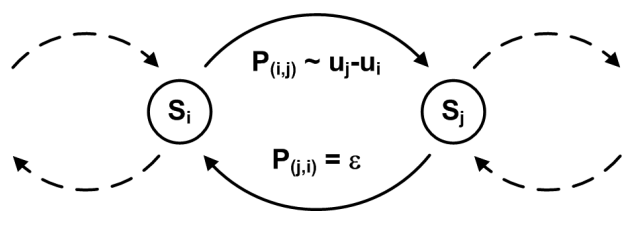

Fig. 5. Parte do modelo em Cadeia de Markov

canal, $B_{i}=22.0 \mathrm{MHz}$ ) ou larga (2 canais, $\left.B_{i}=44 \mathrm{MHz}\right)$. O mesmo vale para a potência, baixa $\left(P_{i}=20 \mathrm{dBm}\right)$ ou alta $\left(P_{i}=13 \mathrm{dBm}\right)$. Por fim, a modulação pode ser QAM com 2 símbolos $\left(M_{i}=2\right)$ ou com 16 símbolos $\left(M_{i}=16\right)$. De acordo com essa descrição, temos um espaço de estratégias com 20 possibilidades distintas.

\section{A. Avaliação com Cadeias de Markov}

Utilizamos uma metodologia semelhante à proposta em [8] para modelar o comportamento dos jogadores com cadeias de Markov. Nesse modelo, os estados da cadeia representam o número de jogadores adotando cada uma das possíveis estratégias. As probabilidades de transição entre estados da cadeia são proporcionais ao aumento obtido na utilidade com a mudança de estratégia. Assim, é representada a motivação de um jogador em alterar sua estratégia sempre visando o de aumento de sua recompensa. Porém, a cadeia de Markov também descreve o fato de um jogador poder cometer erros ao escolher uma estratégia, o que é feito através de uma probabilidade de transição $(\varepsilon)$ muito baixa entre dois estados.

A Figura 5 mostra um exemplo de dois estados desta cadeia. Os estados $S_{i}$ e $S_{j}$ representam quantos jogadores adotam cada uma das possíveis estratégias do jogo, enquanto $P_{(i, j)}$ e $P_{(j, i)}$ representam as probabilidade de transição do estado $S_{i}$ para o $S_{j}$ e vice-versa. Como o retorno do jogador que mudou de estratégia levando o jogo do estado $S_{i}$ para o $S_{j}$ é maior no segundo estado $\left(u_{i}<u_{j}\right)$, então $P_{(i, j)}$ é proporcional à diferença $u_{j}-u_{i}$ e $P_{(j, i)}$ é igual a um $\varepsilon$ muito pequeno, referente ao aprendizado do jogador.

A cadeia de Markov descreve uma dinâmica populacional dos jogadores, ou seja, quantos estão em cada estratégia. Enquanto as transições são obtidas a partir de um modelo de desempenho subjacente. Em nosso trabalho, esse modelo corresponde ao descrito na Seção IV-B, o qual é diferente do modelo utilizado em [8].

Uma das dificuldades que encontramos no uso da modelagem por cadeia de Markov foi o número de estados, o qual motivou o uso de um cenário simples. O problema é que a quantidade de estados aumenta muito com o número de estratégias. Considerando que temos apenas 2 jogadores utilizando o modelo inicial com 20 estratégias, obtemos uma cadeia com 210 estados. Para ilustrar o crescimento do número de estados, se desejássemos aumentar o número de jogadores, mantendo as mesmas 20 estratégias, teríamos uma cadeia de 20.030.010 estados com 10 jogadores. Esse aumento limita o uso dessa abordagem a cenários muito simples, pois um número muito alto de estados torna inviável computacionalmente a montagem da cadeia e a sua solução.

Para solucionar o modelo do jogo utilizando cadeias de Markov, desenvolvemos um programa em linguagem C capaz 
de montar a cadeia em questão, determinando seus estados e transições válidas. O programa identifica também os estados de interesse: equilíbrio de Nash e o ótimo de Pareto. Para ilustrar o problema da complexidade computacional citado anteriormente, com o aumento do número de jogadores o tempo de execução do programa passa de centésimos de segundo no caso de 2 jogadores, para mais de 2 horas e meia de execução no caso de 3 jogadores. Por essa razão, os resultados utilizando cadeias de Markov ficaram limitados à 2 e 3 jogadores no cenário com 20 estratégias.

$\mathrm{O}$ resultado para o caso de 2 e 3 jogadores mostrou um comportamento que já era esperado. Em ambos os casos, os equilíbrios de Nash e ótimos de Pareto foram iguais, e eram atingidos em estados onde não existia nenhum tipo de sobreposição espectral entre os jogadores e todos utilizavam potência alta e modulação 16-QAM. Estes resultados são justificados pelo número de jogadores ser menor ou igual ao número de canais disponíveis para o uso no cenário em avaliação. Portanto, sempre é possível que um jogador mude individualmente para uma estratégia onde não sofra interferência, aumentando sua recompensa. Ao mesmo tempo em que faz isso, ele também poupa seus adversários da sua própria interferência, aumentando a utilidade destes também. Quando uma estratégia onde não existe interferência é alcançada, nenhum jogador consegue aumentar sua utilidade.

\section{B. Avaliação com Algoritmos Genéticos}

Foram repetidas as avaliações da seção anterior, com 2 e 3 jogadores, utilizando o modelo utilizando algoritmos genéticos. Nos resultados obtidos persiste o ganho com o não-compartilhamento de canal, a adoção da potência alta e modulação mais complexa.

Aumentou-se então o número de jogadores para 4. Nesse cenário, existem importantes alterações no desempenho final do sistema, considerando-se que certamente há compartilhamento de canal entre jogadores. O ponto ótimo de Pareto apresenta 3 dos 4 jogadores transmitindo com potência máxima e modulação 16-QAM. O quarto jogador transmite em um único canal, com potência baixa e modulação 2-QAM.

Por fim, foi feita uma avaliação com 10 usuários. Nessa simulação, dada a dimensão do espaço de estratégias, são verificadas 12000 gerações do modelo, cada uma com 120 indivíduos. Um aspecto a ressaltar é o tempo de duração da simulação em torno de 24 segundos, mostrando a vantagem desta abordagem em relação à de cadeia de Markov. No ponto ótimo de Pareto, a simulação apresenta 2 dos 10 jogadores transmitindo com potência máxima e modulação 16-QAM. Os outros 8 jogadores transmitem em um só canal, com potência alta e modulação 2-QAM, certamente para minimizar a taxa de erro de bits.

Considerando a avaliação feita com cadeias de Markov e algoritmos genéticos, observamos que as respostas encontradas indicam que os benefícios obtidos pelos jogadores que ocupam canais sem interferência mais que compensam os prejuízos dos demais. Desta forma, percebe-se que, para permitir um desempenho melhor ou mais justo, mecanismos de punição devem ser estabelecidos, de sorte que o sistema evolua para um equilíbrio estável de acordo com uma distribuição mais uniforme do espectro disponível.

\section{Conclus ão e Trabalhos Futuros}

Este trabalho avaliou duas técnicas de modelagem e resolução do problema do compartilhamento espectral utilizando teoria dos jogos evolucionária. Com cadeias de Markov foi possível solucionar o jogo determinando exatamente os seus equilíbrios de Nash e ótimos de Pareto. Porém, o tempo computacional se torna muito alto devido ao crescimento excessivo do número de estados da cadeia. Por outro lado, os algoritmos genéticos conseguiram identificar ótimos de Pareto rapidamente e, dependendo do modelo de representação do jogo, podem também obter os equilíbrios de Nash.

Outra característica interessante verificada foi que os equilíbrios de Nash e ótimos de Pareto foram sempre os mesmos. Este fato mostra que ao modelar o problema do compartilhamento espectral meramente do ponto de vista técnico utilizando dinâmicas populacionais simples, o jogo converge para equilíbrios interessantes onde não existem maiores ganhos globais. Esta característica fornecerá uma intuição interessante para o prosseguimento deste trabalho, onde uma das metas é o desenvolvimento de mecanismos para o controle do compartilhamento espectral entre dispositivos cognitivos.

Entretanto, maiores estudos serão necessários para avaliar como outras características não presentes neste nosso modelo inicial afetam a sua convergência. Com por exemplo, usuários com diferentes interesses (aplicações heterogêneas), diferentes tipos de usuários (maliciosos e cooperadores) ou outros níveis de modelagem (novas métricas para utilidade).

\section{REFERÊNCIAS}

[1] I. F. Akyildiz, W. Y. Lee, M. C. Vuran, and S. Mohanty. NeXt Generation / Dynamic Spectrum Access / Cognitive Radio Wireless Networks: A Survey. Computer Networks Journal (Elsevier), September 2006.

[2] D. Cabric, S. Mishra, and R. Brodersen. Implementation issues in spectrum sensing for cognitive radios. In Conference on Signals, Systems and Computers, November 2004.

[3] C. Cordeiro, K. Challapali, D. Birru, and S. Shankar. IEEE 802.22: The first worldwide wireless standard based on cognitive radios. In IEEE DySPAN, 2005.

[4] D. E. Goldberg. Genetic algorithms in search, optimization, and machine learning. Addison-Wesley, Reading, MA, 1989.

[5] Zhu Han, Charles Pandana, and K. J. Ray Liu. Distributive Opportunistic Spectrum Access for Cognitive Radio using Correlated Equilibrium and No-Regret Learning. In Wireless Communications and Networking Conference (WCNC), March 2007.

[6] S. Haykin. Cognitive radio: Brain-empowered wireless communications. In Journal on Selected Areas in Communications, February 2005.

[7] S. Mangold, Z. Zhong, K. Challapali, and C.-T. Chou. Spectrum agile radio: Radio resource measurements for opportunistic spectrum usage. In IEEE Globecom, November 2004.

[8] Daniel Sadoc Menasché. Controle de congestionamento: Uma abordagem dinâmico-populacional via teoria dos jogos evolucionários, April 2005.

[9] Nie Nie and Cristina Comaniciu. Adaptive Channel Allocation Spectrum Etiquette for Cognitive Radio Networks. In Mobile Networks and Applications, December 2006.

[10] Dusit Niyato and Ekram Hossain. A Game-Theoretic Approsch to Competitive Spectrum Sharing in Cognitive Radio Networks. In Wireless Communications and Networking Conference (WCNC), March 2007.

[11] J. Walko. Cognitive Radio. IEE Review, 51(5):34-37, May 2005. 